\title{
Mitochondrial unfolded protein response transcription factor ATFS-1 increases resistance to exogenous stressors through upregulation of multiple stress response pathways
}

Sonja K. Soo ${ }^{1,2}$, Annika Traa ${ }^{1,2}$, Meeta Mistry ${ }^{3}$, Jeremy M. Van Raamsdonk ${ }^{1,2,4,5}$

${ }^{1}$ Department of Neurology and Neurosurgery, McGill University, Montreal, Quebec, Canada

${ }^{2}$ Metabolic Disorders and Complications Program, and Brain Repair and Integrative

Neuroscience Program, Research Institute of the McGill University Health Centre, Montreal, Quebec, Canada

${ }^{3}$ Bioinformatics Core, Harvard School of Public Health, Harvard Medical School, Boston. MA, USA

${ }^{4}$ Division of Experimental Medicine, Department of Medicine, McGill University, Montreal, Quebec, Canada

${ }^{5}$ Department of Genetics, Harvard Medical School, Boston, MA, USA

Short title: ATFS-1 promotes stress resistance

\section{Corresponding Author:}

Jeremy M. Van Raamsdonk

Research Institute of the McGill University Health Centre 1001 Decarie Blvd., Montreal, Quebec, Canada

1-514-934-1934 ext.76157, jeremy.vanraamsdonk@mcgill.ca 


\section{Abstract}

3 The mitochondrial unfolded protein response (mitoUPR) is an evolutionarily conserved pathway

4 that responds to various insults to the mitochondria through transcriptional changes that

5 restore mitochondrial homeostasis in order to facilitate cell survival. Gene expression changes

6 resulting from the activation of the mitoUPR are mediated by the transcription factor ATFS-

7 1/ATF-5. To further define the mechanisms through which the mitoUPR protects the cell during

8 mitochondrial dysfunction, we characterized the role of ATFS-1 in responding to organismal

9 stress. We found that activation of ATFS-1 is sufficient to cause upregulation of genes involved

10 in multiple stress response pathways, including the DAF-16-mediated stress response pathway,

11 the SKN-1-mediated oxidative stress response pathway, the HIF-mediated hypoxia response

12 pathway, the p38-mediated innate immune response pathway, and antioxidant genes.

13 Moreover, ATFS-1 is required for the upregulation of stress response genes after exposure to

14 exogenous stressors, especially oxidative stress and bacterial pathogens. Constitutive activation

15 of ATFS-1 increases resistance to multiple acute exogenous stressors, while disruption of atfs-1

16 decreases stress resistance. Although ATFS-1-dependent genes are upregulated in multiple

17 long-lived mutants, constitutive activation of ATFS-1 in wild-type animals results in decreased

18 lifespan. Overall, our work demonstrates that ATFS-1 serves a vital role in organismal survival of 19 acute stresses through its ability to activate multiple stress response pathways, but that chronic 20 ATFS-1 activation is detrimental for longevity.

23 Keywords: Aging; Mitochondria; Mitochondrial unfolded protein response; ATFS-1; Stress

24 resistance; $C$. elegans; Genetics; Lifespan; RNA sequencing 
Introduction

The mitochondrial unfolded protein response (mitoUPR) is a stress response pathway that acts to reestablish mitochondrial homeostasis through inducing transcriptional changes of genes involved in metabolism and restoration of mitochondrial protein folding [1]. Various perturbations to the mitochondria can activate mitoUPR, including excess reactive oxygen species (ROS) and defects in mitochondrial import machinery [2]. The mitoUPR is mediated by the transcription factor ATFS-1 (activating transcription factor associated with stress-1) in $C$. elegans [3], or ATF5 in mammals [4].

ATFS-1/ATF5 regulates mitoUPR through its dual targeting domains, a mitochondrial targeting sequence (MTS) and a nuclear localization signal (NLS). Under normal unstressed conditions, the MTS causes ATFS-1 to enter the mitochondria through the HAF-1 import channel. Inside the mitochondria, ATFS-1 is degraded by the Lon protease CLPP-1/CLP1 [3]. However, mitochondrial stress disrupts ATFS-1 import into the mitochondria, resulting in cytoplasmic accumulation of ATFS-1. The NLS of the cytoplasmic ATFS-1 then targets it to the nucleus, where ATFS-1 acts with the transcription factor DVE-1 and transcriptional regulator UBL-5 to upregulate expression of chaperones, proteases, and other proteins [5].

In order to study the role of the mitoUPR in longevity, we previously disrupted atfs-1 in long-lived nuo-6 mutants, which contain a point mutation that affects Complex I of the electron transport chain [6]. nuo-6 mutants have a mild impairment of mitochondrial function that leads to increased lifespan and enhanced resistance to multiple stressors. We found that loss of atfs1 not only decreased the lifespan of nuo- 6 worms, but also abolished the increased stress resistance of these worms, thereby suggesting that ATFS-1 contributes to both longevity and stress resistance in these worms [7].

While a role for the mitoUPR in longevity has been reported [8-11], and debated [12, 
54 found that activation of ATFS- 1 can increase organismal resistance to the pathogenic bacteria $P$.

55 aeruginosa [14], while Pena et al. showed that ATFS-1 activation can protect against anoxia-

56 reperfusion-induced death [15].

57

In this study, we use C. elegans to define the relationship between ATFS-1 and

59 organismal stress resistance, and explore the underlying mechanisms. We find that activation of

60 ATFS-1 is sufficient to upregulate genes from multiple stress response pathways and is

61 important for transcriptional changes induced by oxidative stress and bacterial pathogen

62 exposure. Constitutive activation of ATFS-1 is also sufficient to increase resistance to multiple

63 stressors. While ATFS-1-dependent genes are upregulated in several long-lived mutants

64 representative of multiple pathways of lifespan extension, chronic activation of ATFS-1 does not

65 extend longevity. Overall, our results demonstrate a crucial role for ATFS-1 in organismal stress

66 response through activation of multiple stress response pathways. 


\section{Results}

\section{ATFS-1 activates genes from multiple stress response pathways}

Mild impairment of mitochondrial function through a mutation in nuo- 6 results in the activation of the mitoUPR. We previously performed a bioinformatic analysis of genes that are upregulated in nuo-6 mutants in an ATFS-1-dependent manner, and discovered an enrichment for genes associated with the GO term "response to stress" [7]. Based on this observation, we hypothesized that ATFS-1 may be able to activate other stress response pathways. To test this hypothesis, we quantified the expression of established target genes from eight different stress response pathways under conditions where ATFS-1 is either activated, or where ATFS-1 is disrupted.

To activate ATFS-1, we used the nuo- 6 mutation. We also examined gene expression in two different gain-of-function (GOF) mutants with constitutively active ATFS-1: atfs-1(et15) and atfs-1(et17). Both of these constitutively active ATFS-1 mutants have mutations in the MTS causing increased nuclear localization of ATFS-1 [16]. We used a loss-of-function (LOF) atfs-1 deletion mutation ( $g k 3094$ ) to disrupt ATFS-1 function in wild-type and nuo-6 mutants.

Expression of specific stress response target genes were examined: $h s p-6$ in the mitochondrial unfolded protein response (mitoUPR) pathway; $h s p-4$ in the endoplasmic reticulum unfolded protein response (ER-UPR) pathway; $h s p-16.2$ in the cytoplasmic unfolded protein response pathway (cytoUPR); sod-3 in the DAF-16-mediated stress response pathway; gst-4 in the SKN-1-mediated stress response pathway; nhr-57 in the HIF-1-mediated hypoxia response pathway; Y9C9A.8 in the p38-mediated innate immunity pathway; and trx-2, an antioxidant gene (Table S1). 
(Fig. 1). This indicates that ATFS-1 is not required for the basal expression levels of these stress response genes.

97
Activation of the mitoUPR through mutation of nuo-6 resulted in significant upregulation of target genes from the mitoUPR (hsp-6; Fig. 1A), the DAF-16-mediated stress response (sod-3; Fig. 1D), the SKN-1-mediated oxidative stress response (gst-4; Fig. 1E), the HIF1-mediated hypoxia response (nhr-57; Fig. 1F), the p38-mediated innate immunity pathway (Y9C9A.8; Fig. 1G), and antioxidant defense (trx-2; Fig. 1H). Importantly, for all of these genes, inhibiting the mitoUPR through deletion of atfs-1 prevented the upregulation of the stress response in nuo-6; atfs-1 (gk3094) worms (Fig. 1A, D-H), indicating that ATFS-1 is required for the activation of these stress pathway genes during mitochondrial stress.

Constitutive activation of ATFS-1 in atfs-1(et 15) and atfs-1(et17) mutants resulted in upregulation of most of the same genes that are upregulated in nuo- 6 mutants, except for gst-4 in the SKN-1 pathway (Fig. 1A, D-H). This indicates that ATFS-1 activation is sufficient to induce upregulation of specific stress response genes independently of mitochondrial stress. Activating the mitoUPR through the nuo-6 mutation, or through the constitutively-active ATFS-1 mutants did not significantly increase the expression of target genes from the ER-UPR (hsp-4; Fig. 1B) or the cyto-UPR (hsp-16.2; Fig. 1C).

To gain a more comprehensive view of the extent to which mitoUPR activation causes upregulation of genes in other stress response pathways, we compared genes upregulated in the constitutively active atfs-1 mutant, atfs-1(et15), to genes upregulated by activation of different stress response pathways. As a proof-of-principle, we first examined the overlap between upregulated genes in atfs-1(et15) mutants and genes upregulated by activation of the mitoUPR with spg-7 RNAi in an ATFS-1-dependent manner [3].

We identified genes upregulated by the activation of other stress response pathways from published gene expression studies, and the genes and relevant pathways are listed in 
124 Table S3. Target genes from the ER-UPR pathway were defined as genes upregulated by

125

126

127

128

129

130

131

132

133

134

135

136 tunicamycin exposure and dependent on either ire-1, xbp-1, pek-1 or atf-6 [17]. Cyto-UPR pathway genes are genes upregulated by overexpression of heat shock factor 1 (HSF-1) and genes bound by HSF-1 after a thirty-minute heat shock at $34^{\circ} \mathrm{C}[18,19]$. DAF-16 pathway genes were identified by Tepper et al. by performing a meta-analysis of 46 previous gene expression studies, comparing conditions in which DAF-16 is activated (e.g. daf-2 mutants) and conditions in which the activation is inhibited by disruption of daf-16 (e.g. daf-2;daf-16 mutants) [20]. SKN1 pathway genes were identified as genes that exhibit decreased expression after skn-1 RNAi in wild-type worms, genes that are upregulated in glp-1 mutants in a SKN-1-dependent manner, genes that are upregulated by germline stem cell removal in a SKN-1-dependent manner [21], and genes upregulated in daf-2 mutants in a SKN-1-dependent manner [22]. HIF-1-mediated hypoxia genes are genes induced by hypoxia in a HIF-1-dependent manner [23]. Innate immunity genes are defined as genes upregulated by exposure to Pseudomonas aeruginosa strain PA14 in a PMK-1- and ATF-7-dependent manner [24], where PMK-1 and ATF-7 are part of the p38-mediated innate immune signaling pathway. Finally, antioxidant genes is a comprehensive list of genes involved in antioxidant defense such as superoxide dismutases (sod), catalases $(c t /)$, peroxiredoxins $(p r d x)$, or thioredoxins $(\operatorname{tr} x)$.

In comparing genes upregulated in the constitutively active atfs-1 mutant et15 to these previously published gene lists, we found that $51 \%$ of genes upregulated by $s p g-7$ RNAi in an ATFS-1-dependent manner are also upregulated by constitutive activation of ATFS-1 (Fig. 2A). Similarly, we found a highly significant overlap of upregulated genes between atfs-1(et15) mutants and each of the examined stress response pathways. We found that atfs-1(et15) had a 25\% overlap with genes of ER-UPR pathway (Fig. 2B); 22\% overlap with genes of the Cyto-UPR pathway (Fig. 2C); 26\% overlap with genes of the DAF-16-mediated stress pathway (Fig. 2D); $30 \%$ overlap with genes of the SKN-1-mediated stress pathway (Fig. 2E); $23 \%$ overlap with genes of the HIF-1-mediated hypoxia pathway (Fig. 2F); $22 \%$ overlap with genes of the p38mediated innate immunity pathway (Fig. 2G); and 33\% overlap with antioxidant genes (Fig. 2H). 
152 Combined, this indicates that activation of ATFS-1 is sufficient to upregulate genes in multiple

153 stress response pathways.

\section{ATFS-1 is required for transcriptional responses to exogenous stressors}

156 Having shown that constitutive activation of ATFS-1 can induce upregulation of genes involved 157 in various stress response pathways, we next sought to determine the role of ATFS-1 in the 158 genetic response to different stressors. To do this, we exposed wild-type animals and atfs-1 159 loss-of-function mutants (atfs-1(gk3094)) to six different types of stress and quantified the 160 resulting upregulation of stress response genes using quantitative RT-PCR (qPCR). We found 161 that exposure to either oxidative stress (4 mM paraquat, 48 hours) or the bacterial pathogen

162 Pseudomonas aeruginosa strain PA14 induced a significant upregulation of stress response 163 genes in wild-type worms, but that this upregulation was suppressed by disruption of atfs-1

164 (Fig. 3A,B). In contrast, exposure to heat stress $\left(35^{\circ} \mathrm{C}, 2\right.$ hours; Fig. $3 \mathrm{C}$ ), osmotic stress (300 mM $165 \mathrm{NaCl}, 24$ hours; Fig. 3D), anoxic stress (24 hours; Fig. 3E), or ER stress (tunicamycin for 24 hours;

166 Fig. 3F) caused upregulation of stress response genes in both wild-type and atfs-1(gk3094)

167 worms to a similar extent. Combined, these results indicate that ATFS-1 is required for 168 upregulating stress response genes in response to exposure to oxidative stress or bacterial pathogens.

\section{Modulation of ATFS-1 levels affects resistance to multiple stressors}

172 Due to the crucial role of ATFS-1 in upregulating genes in multiple stress response pathways, we

173 next sought to determine the extent to which activating ATFS-1 protects against exogenous

174 stressors. To do this, we quantified resistance to stress in two constitutively active atfs-1 gain-

175 of-function mutants (atfs-1(et15), atfs-1(et17)) compared to wild-type worms. For comparison, 176 we also included an atfs-1 loss-of-function deletion mutant (atfs-1(gk3094)), which we have 177 previously shown to have decreased resistance to oxidative, heat, osmotic and anoxic stress [7]. 
181 increased resistance to acute oxidative stress compared to wild-type worms, while atfsresistance to chronic oxidative stress, worms were transferred to plates containing $4 \mathrm{mM}$ paraquat beginning at day 1 of adulthood. Similar to the acute assay, atfs-1(et17) mutants were more resistant to chronic oxidative stress, while atfs-1(gk3094) mutants were less resistant to chronic oxidative stress compared to wild-type worms (Fig. 4B). Oddly, atfs-1(et15) gain-offunction mutants exhibited decreased resistance to chronic oxidative stress.

Resistance to heat stress was measured at $37^{\circ} \mathrm{C}$. None of the mutants showed increased survival during heat stress, with atfs-1(et15) and atfs-1(gk3094) mutants both showing a significant decrease in survival compared to wild-type worms (Fig. 4C). Resistance to osmotic stress was quantified on plates containing $450 \mathrm{mM}$ or $500 \mathrm{mM} \mathrm{NaCl}$ after 48 hours. At both concentrations, the constitutively active atfs-1 mutants had increased survival compared to wild-type worms, while atfs-1(gk3094) deletion mutants had decreased survival, although the difference was only significant at $500 \mathrm{mM}$ (Fig. 4D, E). Resistance to anoxic stress was measured by placing worms in an oxygen-free environment for 75 hours, followed by a 24-hour recovery. We observed increased survival in atfs-1(et15) and atfs-1(et17) mutants and decreased survival in atfs-1(gk3094) mutant compared to wild-type worms (Fig. 4F). aeruginosa strain PA14 in either a fast kill assay in which worms die from a toxin produced by the bacteria, or a slow kill assay in which worms die due to the intestinal colonization of the pathogenic bacteria [25]. In the fast kill assay, we found that constitutive activation of ATFS-1 increases survival in atfs-1(et15) and atfs-1(et17) mutants (Fig. 4G). We also observed increased survival in atfs-1(gk3094) deletion mutants. For the slow kill assay, we used two established protocols: one in which the assay is initiated at the L4 larval stage and performed at $25^{\circ} \mathrm{C}[14$, 20725,26 ] and one in which the assay is initiated at day three of adulthood and performed at $20^{\circ} \mathrm{C}$ 208 [27]. Surprisingly, at $25^{\circ} \mathrm{C}$, we found that atfs-1(et17) mutant had a small decrease in resistance 
compared to wild-type worms (Fig. $4 \mathrm{H})$. At $20^{\circ} \mathrm{C}$, both atfs-1(gk3094) and atfs-1(et17) mutants had a small increase in resistance to PA14 compared to wild-type worms (Fig. I).

All together, these data indicate that activation of ATFS-1 is sufficient to protect against oxidative stress, osmotic stress, anoxia, and bacterial pathogens but not heat stress. They also show that ATFS-1 is required for wild-type worms to survive oxidative stress, heat stress, osmotic stress, and anoxia.

\section{Long-lived genetic mutants have upregulation of ATFS-1 target genes}

We previously showed that ATFS-1 target genes are upregulated in three long-lived mitochondrial mutants: clk-1, isp-1 and nuo-6 [6, 7, 28, 29]. To determine if ATFS-1 target genes are specifically upregulated in long-lived mitochondrial mutants, or if they are also upregulated in other mutants with extended longevity, we compared gene expression in six additional longlived mutants, which act through other longevity-promoting pathways, to genes that are upregulated by ATFS-1 activation. These long-lived mutants included sod-2 mutants, which act through increasing mitochondrial reactive oxygen species (ROS) [30]; daf-2 mutants, which have reduced insulin-IGF1 signaling [31]; glp-1 mutants, which have germline ablation [32]; ife-2 mutants, which have reduced translation [33]; osm-5 with reduced chemosensation [34]; and eat-2 with dietary restriction [35].

After identifying genes that are differentially expressed in each of these long-lived mutants, we compared the differentially expressed genes to genes that are upregulated by ATFS-1 activation. We defined ATFS-1-upregulated genes in two ways: (1) genes that are upregulated by spg-7 RNAi in an ATFS-1-dependent manner [3]; and (2) genes that are upregulated in a constitutively active atfs-1 mutant (et15; [7]).

We found that the majority of the long-lived mutants examined had a significant enrichment of ATFS-1 target genes. In comparing the number of overlapping genes between genes upregulated in each long-lived mutant and genes upregulated by spg-7 RNAi in an ATFS- 
1-dependent manner, the degree of overlap was significantly greater than would be expected by chance for clk-1 (6.7 fold enrichment), isp-1 (6.0 fold enrichment), sod-2 (5.5 fold enrichment), nuo-6 (4.1 fold enrichment), daf-2 (2.6 fold enrichment), glp-1 (2.0 fold enrichment), and ife-2 mutants (1.5 fold enrichment)(Fig. 5). We did not find a significant enrichment of ATFS-1 targets in osm-5 and eat-2 worms (Fig. 5).

In comparing the number of overlapping genes between genes upregulated in each long-lived mutant and genes upregulated in the constitutively active atfs-1(et15) mutant, we found that the degree of overlap was significantly greater than would be expected by chance for isp-1 (3.5 fold enrichment), sod-2 (3.4 fold enrichment), clk-1 (3.3 fold enrichment), nuo-6 (2.5 fold enrichment), daf-2 (2.4 fold enrichment), glp-1 (1.8 fold enrichment), ife-2 (1.8 fold enrichment), and eat-2 mutants (1.5 fold enrichment) (Fig. S1). We did not observe a significant enrichment of ATFS-1 targets in osm-5 mutants (Fig. S1).

Overall, these results indicate that ATFS-1 target genes are upregulated in multiple longlived mutants, including mutants in which mitochondrial function is not directly disrupted.

\section{Constitutively active atfs-1 mutants have decreased lifespan despite enhanced resistance to} stress

Having shown that ATFS-1 target genes are activated in multiple long-lived mutants, we sought to determine if ATFS-1 activation is sufficient to increase lifespan, and whether the presence of ATFS-1 is required for normal longevity in wild-type worms. Despite having increased resistance to multiple stresses, both constitutively active atfs-1 mutants (et15 and et17) have decreased lifespan compared to wild-type worms (Fig. 6A,B), which is consistent with a previous study finding shortened lifespan in atfs-1(et17) and atfs-1(et18) worms [13]. Despite having decreased resistance to multiple stresses, atfs-1 deletion mutants ( $g$ k3094), had a lifespan comparable to wild-type worms (Fig. 6C), as we have previously observed [7]. Combined, this indicates that ATFS-1 does not play a major role in lifespan determination in a wild-type background despite having an important role in stress resistance. 


\section{Discussion}

269

Mitochondria are vital for organismal health as they perform multiple crucial functions within

271 the cell including energy generation, metabolic reactions and intracellular signaling.

272 Accordingly, it is important for cell and organismal survival maintain mitochondrial function

273 during times of acute stress, and throughout normal aging. The mitoUPR is a conserved

274 pathway that facilitates restoration of mitochondrial homeostasis after internal or external

275 stresses. In this work, we demonstrate a crucial role for the mitoUPR transcription factor ATFS-1

276 in the genetic response to external stressors which ultimately promotes survival of the

277 organism.

278

\section{ATFS-1 is not required for normal longevity}

280 A number of studies have directly or indirectly examined the role of the mitoUPR and or ATFS-1

281 in longevity. In these studies, mitoUPR activation was typically measured using a mitoUPR reporter strain that expresses GFP under the promoter of $h s p-6$, which is a target gene of ATFS1 and the mitoUPR.

A relationship between the mitoUPR and longevity was initially supported by the observation that disruption of the mitochondrial electron transport chain (ETC) by RNAi knockdown of the cytochrome $\underline{c}$ oxidase-1 (cco-1) gene resulted in both increased lifespan [36] and activation of the mitoUPR $[8,37]$. Since then, other lifespan-extending mutations have also been shown to activate the mitoUPR, including three long-lived mitochondrial mutants, clk-1, isp-1 and nuo-6 [7].

To explore this relationship in more comprehensive manner, Runkel et al. compiled a list

293 of genes that activate the mitoUPR and looked at their effect on lifespan. Of the 99 genes

294 reported to activated the mitoUPR, 58 resulted in increased lifespan, while only 7 resulted in 295 decreased lifespan [38]. Bennet et al. performed an RNAi screen to identify RNAi clones that 296 increase expression of a reporter of mitoUPR activity ( $h s p-6 p:: G F P)$ and measured the effect of 
a selection of these clones on lifespan [13]. Of the 19 mitoUPR-inducing RNAi clones that they tested, 10 RNAi clones increased lifespan, while 6 decreased lifespan [13]. Using a similar approach to screen for compounds that activate a mitoUPR reporter strain ( $h s p-6 p:: G F P)$, metolazone was identified as a compound that activates the mitoUPR, and extends lifespan in an ATFS-1-dependent manner [39]. Combined these results indicate that there are multiple genes or interventions which activate the mitoUPR and extend longevity, but there are also examples in which these phenotypes are uncoupled.

Multiple experiments including the present study have also looked at the effect of the mitoUPR on lifespan directly by either increasing or decreasing the expression of components of the mitoUPR. Knocking down atfs-1 expression using RNAi does not decrease wild-type lifespan [7, 13, 40], nor do deletions in the atfs-1 gene decrease wild-type lifespan (Fig. 6; [7, 13]). Thus, despite activation of the mitoUPR being correlated with longevity, ATFS-1 is not required for normal lifespan in a wild-type animal.

\section{ATFS-1 mediates lifespan extension in long-lived mutants}

While ATFS-1 is dispensable for wild-type lifespan, ATFS-1 is required for lifespan extension of multiple long-lived mutants. Longevity can be extended by disrupting mito-nuclear protein balance through knocking down the expression of mitochondrial ribosomal protein S5 ( $m r s p-5)$, which also increases the expression of mitoUPR target gene $h s p-6$. The magnitude of the lifespan extension caused by mrsp-5 RNAi is decreased by knocking down key mitoUPR component genes haf-1 or ubl-5 [11]. In the long-lived mitochondrial mutant nuo-6, deletion of atfs-1 completely reverts the long lifespan to wild-type length, and treatment with atfs-1 RNAi has similar effects [7]. In mitochondrial mutant isp-1 worms, knocking down the a key initiatior of mitoUPR, $u b /-5$, decreases their long lifespan but has no effect on the lifespan of wild-type worms [8]. In contrast it has been reported that knockdown of atfs-1 using RNAi does not decrease isp-1 lifespan [13]. However, it is possible that in the latter study that the magnitude knockdown was not sufficient to have effects on lifespan, as we and others have found that lifelong exposure to atfs-1 RNAi prevents larval development of isp-1 worms [7,41]. Similarly, 
326

327

328

329

330

331

332

333

334

335

336

337

338

339

340

341

342

343

344

345

346

347

348

349

350

351

352

353

354

differing results have been obtained for the requirement of the mitoUPR in the extended lifespan resulting from cco-1 knockdown. While it has been reported that mutation of atfs-1 does not decrease lifespan of worms treated with cco-1 RNAi, despite preventing activation of mitoUPR reporter [13], a subsequent study showed that atfs-1 RNAi decreases the extent of lifespan extension resulting from cco-1 RNAi [40]. While differing results have been observed in some cases, overall, these studies suggest a role of ATFS-1 and the mitoUPR in mediating the lifespan extension in a subset of long-lived mutants.

Despite the fact that long-lived mutants with chronic activation of the mitoUPR depend on ATFS-1 for their long lifespan, our current results using the constitutively active atfs-1(et15) and atfs-1(et17) mutants, as well as previous results using constitutively active atfs-1 mutants (et17 and et18) show that constitutive activation of ATFS-1 in wild-type worms results in decreased lifespan [13]. This may be partially due to activation of ATFS-1 increasing the proportion of damaged mtDNA when heteroplasmy exists [42]. Consistent with this finding, overexpression of the mitoUPR target gene $h s p-60$ also leads to a small decrease in lifespan [43]. In contrast, overexpression of a different mitoUPR target gene, $h s p-6$, is sufficient to increase lifespan [44]. It has also been shown that a hypomorphic reduction-of-function mutation allele of $h s p-6$ (mg583) can also increase lifespan, while $h s p-6$ null mutations are thought to be lethal [45]. Combined these results indicate that chronic activation of the mitoUPR is mildly detrimental for lifespan, but that modulation of specific target genes can be beneficial.

\section{ATFS-1 is necessary for stress resistance in wild-type animals}

While ATFS-1 is not required for longevity in wild-type animals, it plays a significant role in protecting animals against exogenous stressors. Disrupting atfs-1 function decreases organismal resistance to oxidative stress, heat stress, osmotic stress, and anoxia (Fig. 4). Additionally, we previously determined that inhibiting atfs-1 in long-lived nuo- 6 worms completely prevented the increased resistance to oxidative stress, osmotic stress and heat stress typically observed in that mutant [7], and that disruption of atfs-1 in Parkinson's disease 
mutants $p d r-1$ and pink-1 decreased their resistance to oxidative stress, osmotic stress, heat stress, and anoxia [46]. Combined, these results demonstrate that ATFS-1 is required for resistance to multiple types of exogenous stressors.

Even though ATFS-1 is required for the upregulation of stress response genes in response to bacterial pathogens (Fig. 3), deletion of atfs-1 (gk3094 mutation) did not impact bacterial pathogen resistance. Similarly, another atfs-1 deletion mutation (tm4919) was found not to affect survival during exposure to $P$. aeruginosa [14]. Knocking down atfs-1 through RNAi inconsistently decreased survival on P. aeruginosa (e.g. Fig.3a versus Fig.3h in [14]). While the effect of atfs-1 disruption on bacterial pathogen resistance was variable, decreasing the expression of a downstream ATFS-1 target gene, $h s p-60$, by RNAi caused a robust decrease in organismal survival on $P$. aeruginosa [43]. As we have previously found that disrupting atfs-1 induces upregulation of other protective cellular pathways [7] and others have observed a similar phenomenon when a mitoUPR downstream target, $h s p-6$, is disrupted [47], it is possible that the upregulation of other stress pathways may compensate for the inhibition of the mitoUPR, ultimately yielding wild-type or increased levels of resistance to bacterial pathogens, and hiding the normal role of the mitoUPR in resistance to bacterial pathogens.

\section{Activation of ATFS-1 enhances resistance to exogenous stressors}

In this work, we show that constitutive activation of ATFS-1 (atfs-1(et15) and atfs-1(et17) mutants) is sufficient to increase resistance to multiple different exogenous stressors, including acute oxidative stress, osmotic stress, anoxia and bacterial pathogens. Previous studies have shown that activating the mitoUPR, either through spg-7 RNAi or through a constitutively active atfs-1(et15) mutant, decreased risk of death after anoxia-reperfusion [15], and that constitutively active atfs-1(et18) mutants also have increased resistance to $P$. aeruginosa [14]. Overexpression of the mitoUPR target gene $h s p-60$ also increases resistance to $P$. aeruginosa [43]. These results support a clear role for ATFS-1 in surviving external stressors. 


\section{ATFS-1 upregulates target genes of multiple stress response pathways}

385 In exploring the mechanism by which ATFS-1 and the mitoUPR modulate stress resistance, we

386 found that activation of ATFS-1, through either a mutation that mildly impairs mitochondrial

387 function (nuo-6) or through a mutation that constitutively activates ATFS-1 (atfs-1(et15)),

388 causes upregulation of genes involved in multiple stress response pathways including the ER-

389 UPR pathway, the Cyto-UPR pathway, the DAF-16-mediated stress response pathway, the SKN-

390 1-mediated oxidative stress response pathway, the HIF-mediated hypoxia response pathway,

391 the p38-mediated innate immune response pathway and antioxidant genes (Fig. 2). These

392 findings are supported by earlier work demonstrating a role for ATFS-1 in upregulating genes

393 involved in innate immunity. Pellegrino et al. reported a 16\% (59/365 genes) overlap between

394 genes upregulated by activation of the mitoUPR through treatment with spg-7 RNAi and genes

395 upregulated by exposure a bacterial pathogen [14]. A connection between the mitoUPR and the

396 innate immunity pathway was also suggested by the finding that overexpression of a mitoUPR

397 downstream target, $h s p-60$, increases expression of three innate immunity genes: T24B8.5,

398 C17H12.8 and K08D8.5 [43]. Our results clearly indicate that the role of ATFS-1 in stress

399 response pathways is not limited to the innate immunity, but extends to multiple stress

400 response pathways, thereby providing a mechanistic basis for the effect of ATFS-1 on resistance

401 to stress. 


\section{Conclusions}

403 The mitoUPR is required for animals to survive exposure to exogenous stressors, and activation

404 of this pathway is sufficient to enhance resistance to stress (Table S4). In addition to

405 upregulating genes involved in restoring mitochondrial homeostasis, the mitoUPR increases

406 stress resistance by upregulating the target genes of multiple stress response pathways.

407 Although increased stress resistance has been associated with long lifespan, and multiple long-

408 lived mutants exhibit activation of the mitoUPR, constitutive activation of ATFS-1 shortens

409 lifespan while increasing resistance to stress, indicating that the role of ATFS-1 in stress

410 resistance can be experimentally dissociated from its role in longevity. Overall, this work

411 highlights the importance of the mitoUPR in not only protecting organisms from internal stress,

412 but also improving organismal survival upon exposure to external stressors. 


\section{Materials and Methods}

Strains

C. elegans strains were obtained from the Caenorhabditis Genetics Center (CGC): N2 (wild-

417 type), nuo-6(qm200), atfs-1(gk3094), nuo-6(qm200);atfs-1(gk3094), atfs-1(et15), atfs-1(et17),

418 ife-2 (ok306), clk-1(qm30), sod-2(ok1030), eat-2(ad1116), osm-5(p813), isp-1(qm150), daf-

419 2(e1370), and $g / p-1(e 2141)$. Strains were maintained at $20^{\circ} \mathrm{C}$ on nematode grown medium

420 (NGM) plates seeded with OP50 E. coli.

\section{Gene expression in response to stress}

423 Stress treatment: Young adult worms were subject to different stress before mRNA was

424 collected. For heat stress, worms were incubated at $35^{\circ} \mathrm{C}$ for 2 hours and $20^{\circ} \mathrm{C}$ for 4 hours. For oxidative stress, worms were transferred to plates containing $4 \mathrm{mM}$ paraquat and $100 \mu \mathrm{M} F \mathrm{FdR}$

426 for 48 hours. For ER stress, worms were transferred to plates containing $5 \mu \mathrm{g} / \mathrm{mL}$ tunicamycin

427 for 24 hours. For osmotic stress, worms were transferred to plates containing $300 \mathrm{mM} \mathrm{NaCl}$ for

42824 hours. For bacterial pathogen stress, worms were transferred to plates seeded with

429 Pseudomonas aeruginosa strain PA14 for 4 hours. For anoxic stress, worms were put in BD Bio-

430 Bag Type A Environmental Chambers (Becton, Dickinson and Company, NJ) for 24 hours and left

431 to recover for 4 hours. For unstressed control conditions, worms were collected at the young

432 adult stage and at Day 1 adult stage. For Day 2 adult control, worms were transferred NGM

433 plates containing $100 \mu \mathrm{M}$ FUdR and collected 2 days later.

RNA isolation: RNA was harvested as described previously [48]. Plates of worms were washed

436 three times using M9 buffer to remove bacteria and resuspended in TRIZOL reagent. Worms

437 were frozen in a dry ice/methanol bath and then thawed three times and left at room

438 temperature for 15 minutes. Chloroform was mixed into the tubes and mixture was left to sit at 439 room temperature for 3 minutes. Tubes were then centrifuged at 12,000 g for 15 minutes at $440 \quad 4^{\circ} \mathrm{C}$. The upper phase containing the RNA was transferred to a new tube, mixed with 441 isopropanol, and allowed to sit at room temperature for 10 minutes. Tubes were centrifuged at 
$44212,000 \mathrm{~g}$ for 10 minutes at $4^{\circ} \mathrm{C}$. The RNA pellet was washed with $75 \%$ ethanol and resuspended 443 in RNAse-free water.

Quantitative RT-PCR: mRNA was converted to cDNA using a High-Capacity cDNA Reverse

446 Transcription kit (Life Technologies/Invitrogen) as described previously [49]. qPCR was

447 performed using a PowerUp SYBR Green Master Mix kit (Applied Biosystems) in a Viia 7 RT-PCR

448 machine from Applied Biosystems. All experiments were performed with least three biological 449 replicates collected from different days. mRNA levels were normalized to act-3 levels and then

450 expressed as a percentage of wild-type. Primer sequences are as follows:

451 gst-4 (CTGAAGCCAACGACTCCATT, GCGTAAGCTTCTTCCTCTGC),

452 hsp-4 (CTCGTGGAATCAACCCTGAC, GACTATCGGCAGCGGTAGAG),

$453 h s p-6$ (CGCTGGAGATAAGATCATCG, TTCACGAAGTCTCTGCATGG),

$454 h s p-16.2$ (CCATCTGAGTCTTCTGAGATTGTT, CTTTCTTTGGCGCTTCAATC),

455 sod-3 (TACTGCTCGCACTGCTTCAA, CATAGTCTGGGCGGACATTT),

456 sod-5 (TTCCACAGGACGTTGTTTCC, ACCATGGAACGTCCGATAAC),

$457 n h r-57$ (GACTCTGTGTGGAGTGATGGAGAG, GTGGCTCTTGGTGTCAATTTCGGG),

458 gcs-1 (CCACCAGATGCTCCAGAAAT, TGCATTTTCAAAGTCGGTC),

459 trx-2 (GTTGATTTCCACGCAGAATG, TGGCGAGAAGAACACTTCCT),

460 Y9C9A.8 (CGGGGATATAACTGATAGAATGG, CAAACTCTCCAGCTTCCAACA),

461 T24B8.5 (TACACTGCTTCAGAGTCGTG, CGACAACCACTTCTAACATCTG),

462 clec-67 (TTTGGCAGTCTACGCTCGTT, CTCCTGGTGTGTCCCATTTT),

463 dod-22 (TCCAGGATACAGAATACGTACAAGA, GCCGTTGATAGTTTCGGTGT),

$464 c k b-2$ (GCATTTATCCGAGACAGCGA, GCTTGCACGTCCAAATCAAC),

$465 a c t-3$ (TGCGACATTGATATCCGTAAGG, GGTGGTTCCTCCGGAAAGAA).

466

\section{RNA sequencing and bioinformatic analysis}

468 RNA sequencing was performed previously $[50,51]$ and raw data is available on NCBI GEO:

469 GSE93724 [51], GSE110984 [7]. Bioinformatic analysis for this study was used to determine 
470 differentially expressed genes and identify the degree and significance of overlaps between

471 genes sets.

472

473 Determining differentially expressed genes: Samples were processed using an RNA-seq pipeline

474 based on the bcbio-nextgen project (https://bcbio-nextgen.readthedocs.org/en/latest/). We

475 examined raw reads for quality issues using FastQC

476 (http://www.bioinformatics.babraham.ac.uk/projects/fastqc/) in order to ensure library

477 generation and sequencing data were suitable for further analysis. If necessary, we used

478 cutadapt http://code.google.com/p/cutadapt/to trim adapter sequences, contaminant

479 sequences such as polyA tails, and low quality sequences from reads. We aligned trimmed

480 reads to the Ensembl build WBcel235 (release 90) of the C. elegans genome using STAR [52].

481 We assessed quality of alignments by checking for evenness of coverage, rRNA content,

482 genomic context of alignments (for example, alignments in known transcripts and introns),

483 complexity and other quality checks. To quantify expression, we used Salmon [53] to find

484 transcript-level abundance estimates and then collapsed down to the gene-level using the $\mathrm{R}$

485 Bioconductor package tximport [54]. Principal components analysis (PCA) and hierarchical

486 clustering methods were used to validate clustering of samples from the same batches

487 and across different mutants. We used the R Bioconductor package DESeq2 [55] to find

488 differential expression at the gene level. For each wildtype-mutant comparison, we identified

489 significant genes using an FDR threshold of 0.01. Lastly, we included batch as a covariate in the

490 linear model for datasets in which experiments were run across two batches.

492 Venn diagrams: Weighted Venn diagrams were produced by inputting gene lists into BioVenn

493 (https://www.biovenn.nl/ ). Percentage overlap was determined by dividing the number of

494 genes in common between the two gene sets by the gene list with the smaller gene list.

Significance of overlap and enrichment: The significance of overlap between two gene sets was

497 determined by comparing the actual number of overlapping genes to the expected number of

498 overlapping genes based on the sizes of the two gene sets (expected number = number of 
genes in set $1 \mathrm{X}$ number of genes in set $2 /$ number of genes in genome detected). Enrichment was calculated as the observed number of overlapping genes/the expected number of overlapping genes if genes were chosen randomly.

\section{Resistance to stress}

For acute oxidative stress, young adult worms were transferred onto plates with $300 \mu \mathrm{M}$ juglone and survival was measured every 2 hours for a total of 10 hours. For chronic oxidative stress, young adult worms were transferred onto plates with $4 \mathrm{mM}$ paraquat and $100 \mu \mathrm{M}$ FUdR and survival was measured daily until death.

For heat stress, young adult worms were incubated in $37^{\circ} \mathrm{C}$ and survival was measured every 2 hours for a total of 10 hours. For osmotic stress, young adult worms were transferred to plates containing $450 \mathrm{mM}$ or $500 \mathrm{mM} \mathrm{NaCl}$ and survival was measured after 48 hours.

For anoxic stress, plates with young adult worms were put into BD Bio-Bag Type A

Environmental Chambers for 75 hours and survival was measured after a 24-hour recovery period.

Two different bacterial pathogenesis assays involving $P$. aeruginosa strain PA14 were performed. In the slow kill assay worms are thought to die from intestinal colonization of the pathogenic bacteria, while in the fast kill assay worms are thought to die from a toxin secreted from the bacteria [25]. The slow kill assay was performed as described previously [14, 27]. In the first protocol [14], PA14 cultures were grown overnight and seeded to center of a 35-mm NGM agar plate. Plates were left to dry overnight, and then incubated in $37^{\circ} \mathrm{C}$ for 24 hours. Plates were left to adjust to room temperature before approximately 40 L 4 worms were transferred onto the plates. The assay was conducted $25^{\circ} \mathrm{C}$ and plates were checked twice a day until death. In the second protocol [27], overnight PA14 culture were seeded to the center of a 35-mm NGM agar plate containing $20 \mathrm{mg} / \mathrm{L} \mathrm{FUdR}$. Plates were incubated at $37^{\circ} \mathrm{C}$ overnight, then at room temperature overnight before approximately 40 day three adults were transferred onto these plates. The assay was conducted $20^{\circ} \mathrm{C}$ and plates were checked daily until death. The fast kill pathogenesis assay was performed as described previously [25]. PA14 
527 cultures were grown overnight and seeded to Peptone-Glucose-Sorbitol (PGS) agar plates.

528 Seeded plates were left to dry for 20 minutes at room temperature before incubation at $37^{\circ} \mathrm{C}$

529 for 24 hours and then at $23^{\circ} \mathrm{C}$ for another 24 hours. Approximately $30 \mathrm{~L} 4$ worms were

530 transferred onto the plates and were scored as dead or alive at 2, 4, 6, 8 and 24 hours. Fast kill

531 plates were kept at $23^{\circ} \mathrm{C}$ in between scoring timepoints.

533 Lifespan

534 All lifespan assays were performed at $20^{\circ} \mathrm{C}$. Lifespan assays included FUdR to limit the

535 development of progeny and the occurrence of internal hatching. Based on our previous

536 studies, a low concentration of FUdR $(25 \mathrm{mM})$ was used to minimize potential effects of FUdR

537 on lifespan [56]. Animals were excluded from the experiment if they crawled off the plate or

538 died of internal hatching of progeny or expulsion of internal organs.

\section{Statistical Analysis}

541 To ensure unbiased results, all experiments were conducted with the experimenter blinded to

542 the genotype of the worms. For all assays, a minimum of three biological replicates of randomly

543 selected worms from independent populations of worms on different days were used. For

544 analysis of lifespan, oxidative stress, and bacterial pathogen stress, a log-rank test was used. For

545 analysis of heat stress, repeated measures ANOVA was used. For analysis of osmotic stress and

546 anoxic stress, a one-way ANOVA with Dunnett's multiple comparisons tests was used. For

547 quantitative PCR results we used a two-way ANOVA with Bonferroni post-test. For all bar

548 graphs error bars indicate standard error of the mean and bars indicate the mean. 
549

550

551

552

553

554

555

556

557

558

559

560

561

562

563

564

565

566

567

568

569

570

571

572

573

574

Acknowledgments. We would like to thank Paige Rudich for carefully reviewing the manuscript prior to submission. Some strains were provided by the CGC, which is funded by NIH Office of Research Infrastructure Programs (P30 OD010440). We would also like to acknowledge the $C$. elegans knockout consortium and the National Bioresource Project of Japan for providing strains used in this research.

Competing interests. The authors have declared that no competing interests exist.

Author Contributions. Conceptualization: JVR. Methodology: SKS, AT, MM, JVR. Investigation: SKS, AT, MM, JVR. Analysis: SKS, AT, MM, JVR. Visualization: SKS, AT, MM, JVR. Writing original draft: SKS, JVR. Writing - review and editing: SKS, AT, JVR. Supervision: JVR.

Materials \& Correspondence. Correspondence and material requests should be addressed to Jeremy Van Raamsdonk.

Data availability. RNA-seq data has been deposited on GEO: GSE93724, GSE110984. All other data and strains generated in the current study are included with the manuscript or available from the corresponding author on request.

Funding. This work was supported by the National Institute of General Medical Sciences (NIGMS; https://www.nigms.nih.gov/; JVR) by grant number R01 GM121756, the Canadian Institutes of Health Research (CIHR; http://www.cihr-irsc.gc.ca/ ; JVR) and the Natural Sciences and Engineering Research Council of Canada (NSERC; https://www.nserc-

72 crsng.gc.ca/index eng.asp; JVR). The funders had no role in study design, data collection and analysis, decision to publish, or preparation of the manuscript. 


\section{References}

1. Zhao Q, Wang J, Levichkin I, Stasinopoulos S, Ryan M, Hoogenraad N. A mitochondrial specific stress response in mammalian cells. The EMBO Journal. 2002;21(17):4411 \pm 9.

2. Shpilka T, Haynes CM. The mitochondrial UPR: mechanisms, physiological functions and implications in ageing. Nat Rev Mol Cell Biol. 2018;19(2):109-20. Epub 2017/11/23. doi: 10.1038/nrm.2017.110. PubMed PMID: 29165426.

3. Nargund AM, Pellegrino MW, Fiorese CJ, Baker BM, Haynes CM. Mitochondrial import efficiency of ATFS-1 regulates mitochondrial UPR activation. Science. 2012;337(6094):587-90. doi: 10.1126/science.1223560. PubMed PMID: 22700657; PubMed Central PMCID: PMC3518298. 4. Fiorese CJ, Schulz AM, Lin YF, Rosin N, Pellegrino MW, Haynes CM. The Transcription Factor ATF5 Mediates a Mammalian Mitochondrial UPR. Curr Biol. 2016. doi: 10.1016/j.cub.2016.06.002. PubMed PMID: 27426517.

5. Jovaisaite V, Mouchiroud L, Auwerx J. The mitochondrial unfolded protein response, a conserved stress response pathway with implications in health and disease. The Journal of experimental biology. 2014;217(Pt 1):137-43. doi: 10.1242/jeb.090738. PubMed PMID: 24353213; PubMed Central PMCID: PMC3867496.

6. Yang W, Hekimi S. Two modes of mitochondrial dysfunction lead independently to lifespan extension in Caenorhabditis elegans. Aging cell. 2010;9(3):433-47. doi: 10.1111/j.14749726.2010.00571.x. PubMed PMID: 20346072.

7. Wu Z, Senchuk MM, Dues DJ, Johnson BK, Cooper JF, Lew L, et al. Mitochondrial unfolded protein response transcription factor ATFS-1 promotes longevity in a long-lived mitochondrial mutant through activation of stress response pathways. BMC biology. 2018;16(1):147. doi: 10.1186/s12915-0180615-3. PubMed PMID: 30563508; PubMed Central PMCID: PMCPMC6298126.

8. Durieux J, Wolff S, Dillin A. The cell-non-autonomous nature of electron transport chainmediated longevity. Cell. 2011;144(1):79-91. doi: 10.1016/j.cell.2010.12.016. PubMed PMID: 21215371; PubMed Central PMCID: PMC3062502.

9. Berendzen KM, Durieux J, Shao LW, Tian Y, Kim HE, Wolff S, et al. Neuroendocrine Coordination of Mitochondrial Stress Signaling and Proteostasis. Cell. 2016;166(6):1553-63 e10. doi: 10.1016/j.cell.2016.08.042. PubMed PMID: 27610575.

10. Merkwirth C, Jovaisaite V, Durieux J, Matilainen O, Jordan SD, Quiros PM, et al. Two Conserved Histone Demethylases Regulate Mitochondrial Stress-Induced Longevity. Cell. 2016. doi: 10.1016/j.cell.2016.04.012. PubMed PMID: 27133168.

11. Houtkooper RH, Mouchiroud L, Ryu D, Moullan N, Katsyuba E, Knott G, et al. Mitonuclear protein imbalance as a conserved longevity mechanism. Nature. 2013;497(7450):451-7. doi: 10.1038/nature12188. PubMed PMID: 23698443; PubMed Central PMCID: PMC3663447. 12. Bennett $\mathrm{CF}$, Kaeberlein $\mathrm{M}$. The mitochondrial unfolded protein response and increased longevity: Cause, consequence, or correlation? Experimental gerontology. 2014. doi: 10.1016/j.exger.2014.02.002. PubMed PMID: 24518875.

13. Bennett CF, Vander Wende H, Simko M, Klum S, Barfield S, Choi H, et al. Activation of the mitochondrial unfolded protein response does not predict longevity in Caenorhabditis elegans. Nature communications. 2014;5:3483. doi: 10.1038/ncomms4483. PubMed PMID: 24662282.

14. Pellegrino MW, Nargund AM, Kirienko NV, Gillis R, Fiorese CJ, Haynes CM. Mitochondrial UPRregulated innate immunity provides resistance to pathogen infection. Nature. 2014;516(7531):414-7. doi: 10.1038/nature13818. PubMed PMID: 25274306; PubMed Central PMCID: PMC4270954. 15. Pena S, Sherman T, Brookes PS, Nehrke K. The Mitochondrial Unfolded Protein Response Protects against Anoxia in Caenorhabditis elegans. PLoS One. 2016;11(7):e0159989. doi: 10.1371/journal.pone.0159989. PubMed PMID: 27459203; PubMed Central PMCID: PMC4961406. 
16. Rauthan M, Ranji P, Aguilera Pradenas N, Pitot C, Pilon M. The mitochondrial unfolded protein response activator ATFS-1 protects cells from inhibition of the mevalonate pathway. Proc Natl Acad Sci U S A. 2013;110(15):5981-6. doi: 10.1073/pnas.1218778110. PubMed PMID: 23530189; PubMed Central PMCID: PMC3625262.

17. Shen X, Ellis RE, Sakaki K, Kaufman RJ. Genetic interactions due to constitutive and inducible gene regulation mediated by the unfolded protein response in C. elegans. PLoS Genet. 2005;1(3):e37. Epub 2005/09/27. doi: 10.1371/journal.pgen.0010037. PubMed PMID: 16184190; PubMed Central PMCID: PMCPMC1231716.

18. Li J, Chauve L, Phelps G, Brielmann RM, Morimoto RI. E2F coregulates an essential HSF developmental program that is distinct from the heat-shock response. Genes Dev. 2016;30(18):2062-75. Epub 2016/11/01. doi: 10.1101/gad.283317.116. PubMed PMID: 27688402; PubMed Central PMCID: PMCPMC5066613.

19. Sural S, Lu TC, Jung SA, Hsu AL. HSB-1 Inhibition and HSF-1 Overexpression Trigger Overlapping Transcriptional Changes To Promote Longevity in Caenorhabditis elegans. G3 (Bethesda). 2019;9(5):1679-92. Epub 2019/03/22. doi: 10.1534/g3.119.400044. PubMed PMID: 30894454; PubMed Central PMCID: PMCPMC6505166.

20. Tepper RG, Ashraf J, Kaletsky R, Kleemann G, Murphy CT, Bussemaker HJ. PQM-1 Complements DAF-16 as a Key Transcriptional Regulator of DAF-2-Mediated Development and Longevity. Cell. 2013;154(3):676-90. doi: 10.1016/j.cell.2013.07.006. PubMed PMID: 23911329.

21. Steinbaugh MJ, Narasimhan SD, Robida-Stubbs S, Moronetti Mazzeo LE, Dreyfuss JM, Hourihan $J M$, et al. Lipid-mediated regulation of SKN-1/Nrf in response to germ cell absence. eLife. 2015;4. doi: 10.7554/elife.07836. PubMed PMID: 26196144.

22. Ewald CY, Landis JN, Porter Abate J, Murphy CT, Blackwell TK. Dauer-independent insulin/IGF-1signalling implicates collagen remodelling in longevity. Nature. 2015;519(7541):97-101. Epub 2014/12/18. doi: 10.1038/nature14021. PubMed PMID: 25517099; PubMed Central PMCID: PMCPMC4352135.

23. Shen C, Nettleton D, Jiang M, Kim SK, Powell-Coffman JA. Roles of the HIF-1 hypoxia-inducible factor during hypoxia response in Caenorhabditis elegans. J Biol Chem. 2005;280(21):20580-8. Epub 2005/03/23. doi: 10.1074/jbc.M501894200. PubMed PMID: 15781453.

24. Fletcher M, Tillman EJ, Butty VL, Levine SS, Kim DH. Global transcriptional regulation of innate immunity by ATF-7 in C. elegans. PLoS Genet. 2019;15(2):e1007830. Epub 2019/02/23. doi: 10.1371/journal.pgen.1007830. PubMed PMID: 30789901; PubMed Central PMCID: PMCPMC6400416. 25. Kirienko NV, Cezairliyan BO, Ausubel FM, Powell JR. Pseudomonas aeruginosa PA14 pathogenesis in Caenorhabditis elegans. Methods Mol Biol. 2014;1149:653-69. doi: 10.1007/978-14939-0473-0_50. PubMed PMID: 24818940.

26. Dues DJ, Andrews EK, Schaar CE, Bergsma AL, Senchuk MM, Van Raamsdonk JM. Aging causes decreased resistance to multiple stresses and a failure to activate specific stress response pathways. Aging (Albany NY). 2016;8(4):777-95. doi: 10.18632/aging.100939. PubMed PMID: 27053445; PubMed Central PMCID: PMC4925828.

27. Wu Z, Isik M, Moroz N, Steinbaugh MJ, Zhang P, Blackwell TK. Dietary Restriction Extends Lifespan through Metabolic Regulation of Innate Immunity. Cell Metab. 2019;29(5):1192-205 e8. Epub 2019/03/25. doi: 10.1016/j.cmet.2019.02.013. PubMed PMID: 30905669; PubMed Central PMCID: PMCPMC6506407.

28. Lakowski B, Hekimi S. Determination of Life-Span in Caenorhabditis elegans by Four Clock Genes. Science. 1996;272:1010-3.

29. Feng J, Bussiere F, Hekimi S. Mitochondrial Electron Transport Is a Key Determinant of Life Span in Caenorhabditis elegans. Developmental Cell. 2001;1:633-64. 
30. Van Raamsdonk JM, Hekimi S. Deletion of the mitochondrial superoxide dismutase sod-2 extends lifespan in Caenorhabditis elegans. PLoS Genet. 2009;5(2):e1000361. doi: 10.1371/journal.pgen.1000361. PubMed PMID: 19197346; PubMed Central PMCID: PMC2628729. 31. Kenyon C, Chang J, Gensch E, Rudner A, Tabtlang R. A C. elegans mutant that lives twice as long as wild type. Nature. 1993;366:461-4.

32. Hsin $\mathrm{H}$, Kenyon $\mathrm{C}$. Signals fromthe reproductive systemregulate the lifespanof $\mathrm{C}$. elegans. Nature. 1999;399:362-6.

33. Hansen M, Taubert S, Crawford D, Libina N, Lee SJ, Kenyon C. Lifespan extension by conditions that inhibit translation in Caenorhabditis elegans. Aging Cell. 2007;6(1):95-110. Epub 2007/02/03. doi: 10.1111/j.1474-9726.2006.00267.x. PubMed PMID: 17266679.

34. Apfeld J, Kenyon C. Regulation of lifespan by sensory perception in Caenorhabditiselegans. Nature. 1999:804-9.

35. Lakowski B, Hekimi S. The genetics of caloric restriction in Caenorhabditis elegans. Proc Natl Acad Sci USA. 1998;95:13091-6.

36. Dillin A, Hsu A, Arantes-Oliveira N, Lehrer-Graiwer J, Hsin H, Fraser A, et al. Rates of Behavior and Aging Specified by Mitochondrial

Function During Development. Science. 2002;298:2398-401.

37. Yoneda T, Benedetti C, Urano F, Clark SG, Harding HP, Ron D. Compartment-specific perturbation of protein handling activates genes encoding mitochondrial chaperones. Journal of cell science. 2004;117(Pt 18):4055-66. doi: 10.1242/jcs.01275. PubMed PMID: 15280428.

38. Runkel ED, Baumeister R, Schulze E. Mitochondrial stress: Balancing friend and foe. Experimental gerontology. 2014. doi: 10.1016/j.exger.2014.02.013. PubMed PMID: 24603155. 39. Ito A, Zhao Q, Tanaka Y, Yasui M, Katayama R, Sun S, et al. Metolazone upregulates mitochondrial chaperones and extends lifespan in Caenorhabditis elegans. Biogerontology. 2021;22(1):119-31. doi: 10.1007/s10522-020-09907-6. PubMed PMID: 33216250.

40. Tian Y, Garcia G, Bian Q, Steffen KK, Joe L, Wolff S, et al. Mitochondrial Stress Induces Chromatin Reorganization to Promote Longevity and UPR(mt). Cell. 2016;165(5):1197-208. doi: 10.1016/j.cell.2016.04.011. PubMed PMID: 27133166; PubMed Central PMCID: PMCPMC4889216. 41. Baker BM, Nargund AM, Sun T, Haynes CM. Protective coupling of mitochondrial function and protein synthesis via the elF2alpha kinase GCN-2. PLoS Genet. 2012;8(6):e1002760. doi: 10.1371/journal.pgen.1002760. PubMed PMID: 22719267; PubMed Central PMCID: PMC3375257. 42. Lin YF, Schulz AM, Pellegrino MW, Lu Y, Shaham S, Haynes CM. Maintenance and propagation of a deleterious mitochondrial genome by the mitochondrial unfolded protein response. Nature. 2016. doi: 10.1038/nature17989. PubMed PMID: 27135930.

43. Jeong DE, Lee D, Hwang SY, Lee Y, Lee JE, Seo M, et al. Mitochondrial chaperone HSP-60 regulates anti-bacterial immunity via p38 MAP kinase signaling. The EMBO journal. 2017;36(8):1046-65. doi: 10.15252/embj.201694781. PubMed PMID: 28283579; PubMed Central PMCID: PMCPMC5391144. 44. Yokoyama K, Fukumoto K, Murakami T, Harada S, Hosono R, Wadhwa R, et al. Extended longevity of Caenorhabditis elegans by knocking in extra copies of hsp70F, a homolog of mot-2 (mortalin)/mthsp70/Grp75. FEBS letters. 2002;516(1-3):53-7. doi: 10.1016/s0014-5793(02)02470-5. PubMed PMID: 11959102.

45. Mao K, Ji F, Breen P, Sewell A, Han M, Sadreyev R, et al. Mitochondrial Dysfunction in C. elegans Activates Mitochondrial Relocalization and Nuclear Hormone Receptor-Dependent Detoxification Genes. Cell metabolism. 2019;29(5):1182-91 e4. doi: 10.1016/j.cmet.2019.01.022. PubMed PMID: 30799287; PubMed Central PMCID: PMCPMC6506380.

46. Cooper JF, Machiela E, Dues DJ, Spielbauer KK, Senchuk MM, Van Raamsdonk JM. Activation of the mitochondrial unfolded protein response promotes longevity and dopamine neuron survival in 
Parkinson's disease models. Scientific reports. 2017;7(1):16441. doi: 10.1038/s41598-017-16637-2. PubMed PMID: 29180793; PubMed Central PMCID: PMC5703891. Coordinates a Mitochondrial-to-Cytosolic Stress Response. Cell. 2016;166(6):1539-52 e16. doi: 10.1016/j.cell.2016.08.027. PubMed PMID: 27610574.

722

48. Schaar CE, Dues DJ, Spielbauer KK, Machiela E, Cooper JF, Senchuk M, et al. Mitochondrial and

723 cytoplasmic ROS have opposing effects on lifespan. PLoS Genet. 2015;11(2):e1004972. doi: 10.1371/journal.pgen.1004972. PubMed PMID: 25671321; PubMed Central PMCID: PMC4335496. 49. Machiela E, Dues DJ, Senchuk MM, Van Raamsdonk JM. Oxidative stress is increased in C. elegans models of Huntington's disease but does not contribute to polyglutamine toxicity phenotypes. Neurobiology of disease. 2016;96:1-11. doi: 10.1016/j.nbd.2016.08.008. PubMed PMID: 27544481. 50. Dues DJ, Schaar CE, Johnson BK, Bowman MJ, Winn ME, Senchuk MM, et al. Uncoupling of oxidative stress resistance and lifespan in long-lived isp-1 mitochondrial mutants in Caenorhabditis elegans. Free radical biology \& medicine. 2017;108:362-73. doi: 10.1016/j.freeradbiomed.2017.04.004. PubMed PMID: 28392283. $16 /$ FOXO by reactive oxygen species contributes to longevity in long-lived mitochondrial mutants in Caenorhabditis elegans. PLoS Genet. 2018;14(3):e1007268. doi: 10.1371/journal.pgen.1007268. PubMed PMID: 29522556; PubMed Central PMCID: PMCPMC5862515.

736 seq aligner. Bioinformatics. 2013;29(1):15-21. doi: 10.1093/bioinformatics/bts635. PubMed PMID: 23104886; PubMed Central PMCID: PMC3530905.

739 53. Patro R, Duggal G, Love MI, Irizarry RA, Kingsford C. Salmon provides fast and bias-aware 740 quantification of transcript expression. Nat Methods. 2017;14(4):417-9. Epub 2017/03/07. doi:

741 10.1038/nmeth.4197. PubMed PMID: 28263959; PubMed Central PMCID: PMCPMC5600148.

742 54. Soneson C, Love MI, Robinson MD. Differential analyses for RNA-seq: transcript-level estimates improve gene-level inferences. F1000Res. 2015;4:1521. Epub 2016/03/01. doi: 10.12688/f1000research.7563.2. PubMed PMID: 26925227; PubMed Central PMCID: PMCPMC4712774. 55. Love MI, Huber W, Anders S. Moderated estimation of fold change and dispersion for RNA-seq data with DESeq2. Genome Biol. 2014;15(12):550. Epub 2014/12/18. doi: 10.1186/s13059-014-0550-8. PubMed PMID: 25516281; PubMed Central PMCID: PMCPMC4302049. 

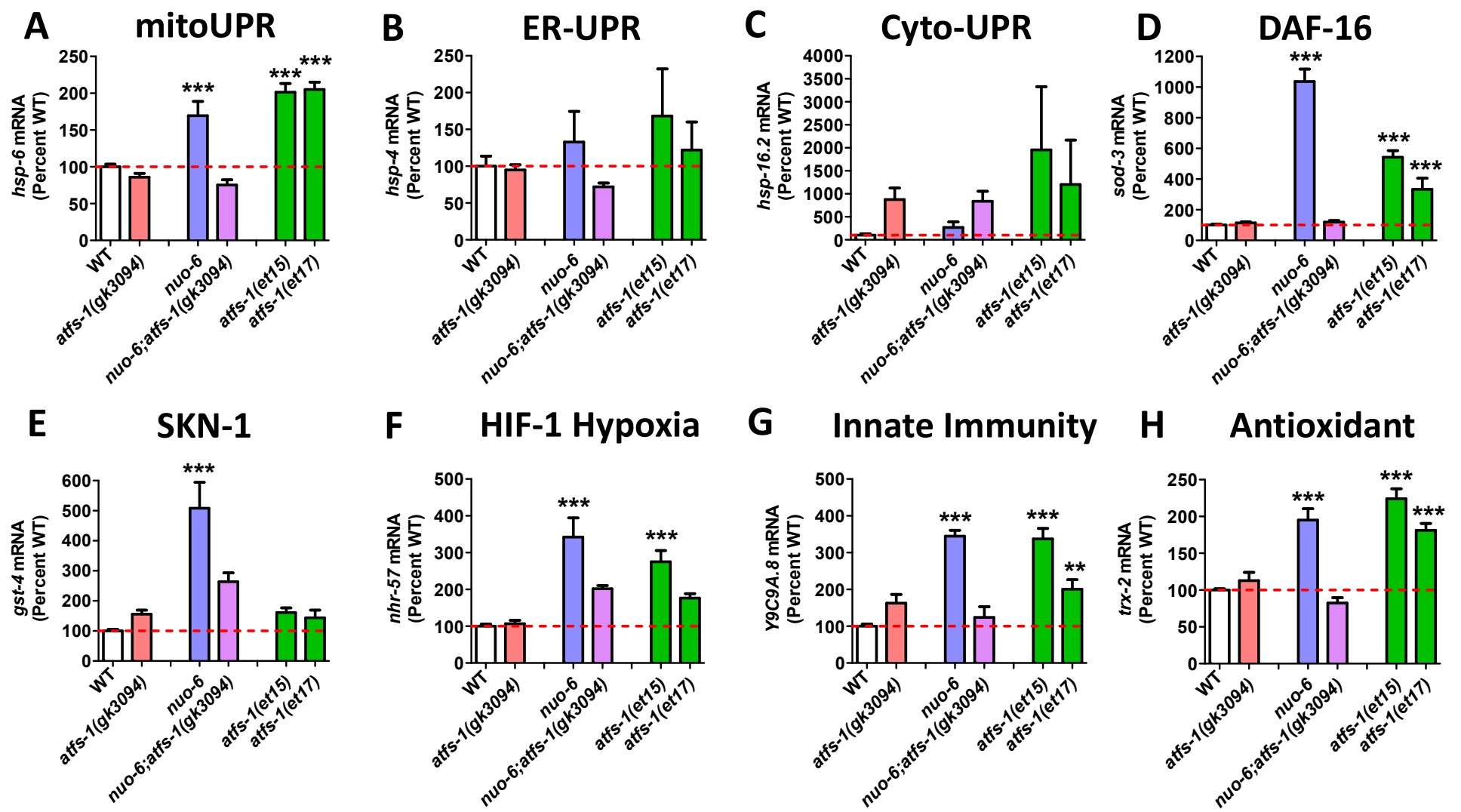

Figure 1. Activation of ATFS-1 upregulates genes from multiple stress response pathways. To determine the role of ATFS-1 in the activation of genes from different stress response pathways, we activated ATFS-1 by mildly impairing mitochondrial function through a mutation in nuo- 6 (blue bars) and then examined the effect of disrupting atfs-1 using an atfs-1 deletion mutant atfs-1 (gk3094) (purple bars). We also examined the expression of these genes in two constitutively active atfs-1 mutants, atfs-1(et15) and atfs-1(et17) (green bars). Target genes from the mitochondrial unfolded protein response (mitoUPR) (A, hsp-6), DAF-16-mediated stress response (D, sod-3), SKN-1-mediated oxidative stress response (E, gst-4), HIF-1-mediated hypoxia response (F, nhr-57), p38-mediated innate immune pathway (G, Y9C9A.8), and antioxidant defense $(\mathbf{H}, t r x-2)$ are all significantly upregulated in nuo- 6 mutants in an ATFS-1-dependent manner. Target genes from the mitoUPR, DAF16-mediated stress response, HIF-1-mediated hypoxia response, p38-mediated innate immune pathway and antioxidant defense are also upregulated in constitutive activation of ATFS-1 (A, D, F, G, H). In contrast, activation of ATFS-1 by nuo- 6 mutation or atfs- 1 gain-of-function mutations did not significantly affect target gene expression for the endoplasmic reticulum unfolded protein response (B, ER-UPR, hsp-4) or the cytoplasmic unfolded protein response (C, Cyto-UPR, hsp-16.2). atfs1 (gk3094) is a loss-of-function deletion mutant. atfs-1(et15) and atfs-1(et17) are constitutively active gain-of-function mutants. Error bars indicate SEM. ${ }^{* *} p<0.01,{ }^{* * *} p<0.001$. A full list of genes that are upregulated by ATFS-1 activation can be found in Table $\mathbf{S 2}$. 


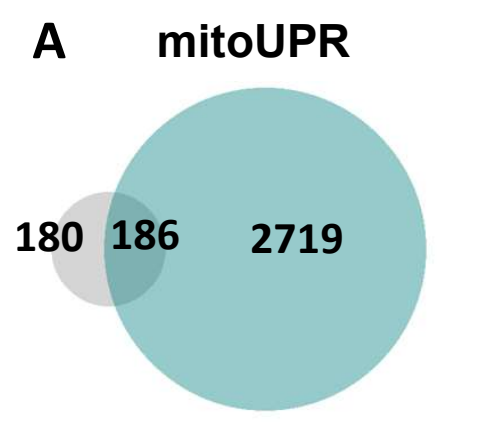

$51 \%$ overlap; $p=4.6 \times 10^{-53}$

E

734308
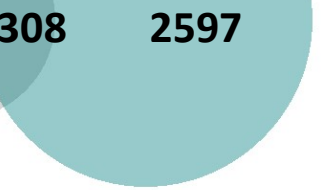

$30 \%$ overlap; $p=7.5 \times 10^{-28}$

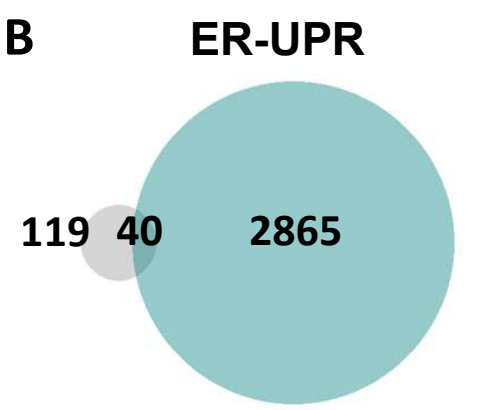

$25 \%$ overlap; $p=0.003$

$\mathbf{F}$

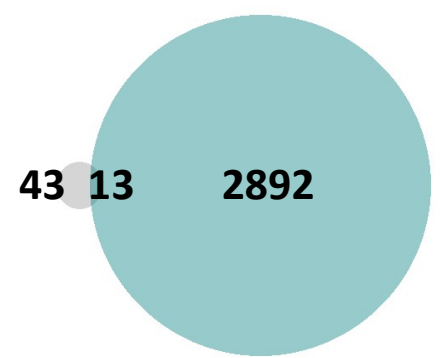

$23 \%$ overlap; $p<0.05$

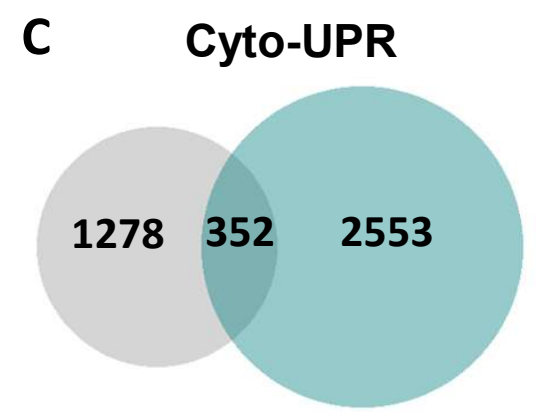

$22 \%$ overlap; $p=1 \times 10^{-8}$
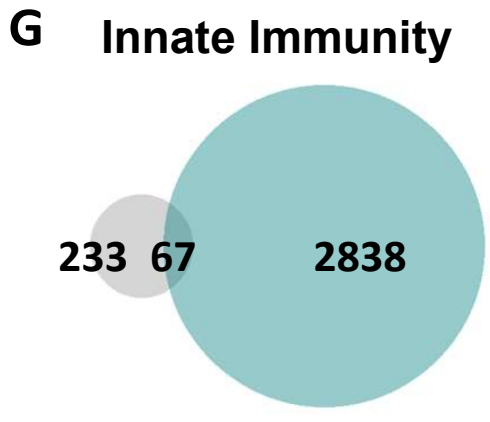

$22 \%$ overlap; $p=0.005$

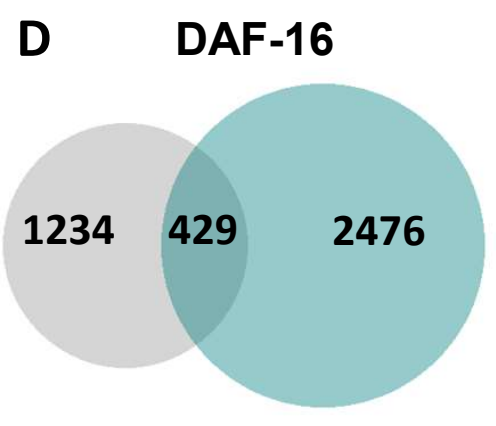

$26 \%$ overlap; $p=9 \times 10^{-25}$

H Antioxidant

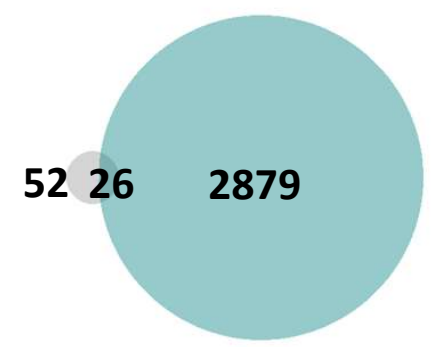

$33 \%$ overlap; $p=2.6 \times 10^{-5}$

Figure 2. Constitutive activation of ATFS-1 results in upregulation of genes from multiple stress response pathways. Genes that are upregulated by activation of ATFS-1 were compared to previous published lists of genes involved in different stress response pathways, including the mitochondrial unfolded protein response (A, mitoUPR), the endoplasmic reticulum unfolded protein response (B, ERUPR), the cytoplasmic unfolded protein response (C, Cyto-UPR), the DAF-16-mediated stress response (D), the SKN-1-mediated oxidative stress response (E), the HIF-1-mediated hypoxia response (F), the p38-mediated innate immune response $(\mathbf{G})$, and antioxidant genes $(\mathbf{H})$. In every case, there was a significant degree of overlap ranging from $22 \%-51 \%$. Grey circles indicate genes that are upregulated by activation of the stress response pathway indicated. Turquoise circles indicate genes that are upregulated in the atfs-1(et15) constitutively active gain-of-function mutant. The numbers inside the circles show how many genes are upregulated. The percentage overlap is the number of overlapping genes as a percentage of the number of genes upregulated by the stress response pathway. mitoUPR = mitochondrial unfolded protein response. ER-UPR = endoplasmic reticulum unfolded protein response. Cyto-UPR = cytoplasmic unfolded protein response. DAF-16 = DAF-16-mediated stress response pathway. SKN-1 = SKN-1-mediated oxidative stress response pathway. HIF-1 = HIF-1-mediated stress response pathway. Innate immunity = p38-mediated innate immunity pathway. Antioxidant $=$ antioxidant genes. Stress pathway gene lists and sources can be found in Table S3. 
A bioRxiv preprint dpi hthtps://doi.org/10.1101/2021.04.26.441408; this version posted April 26, 2021. The copyright holder for this preprint (which
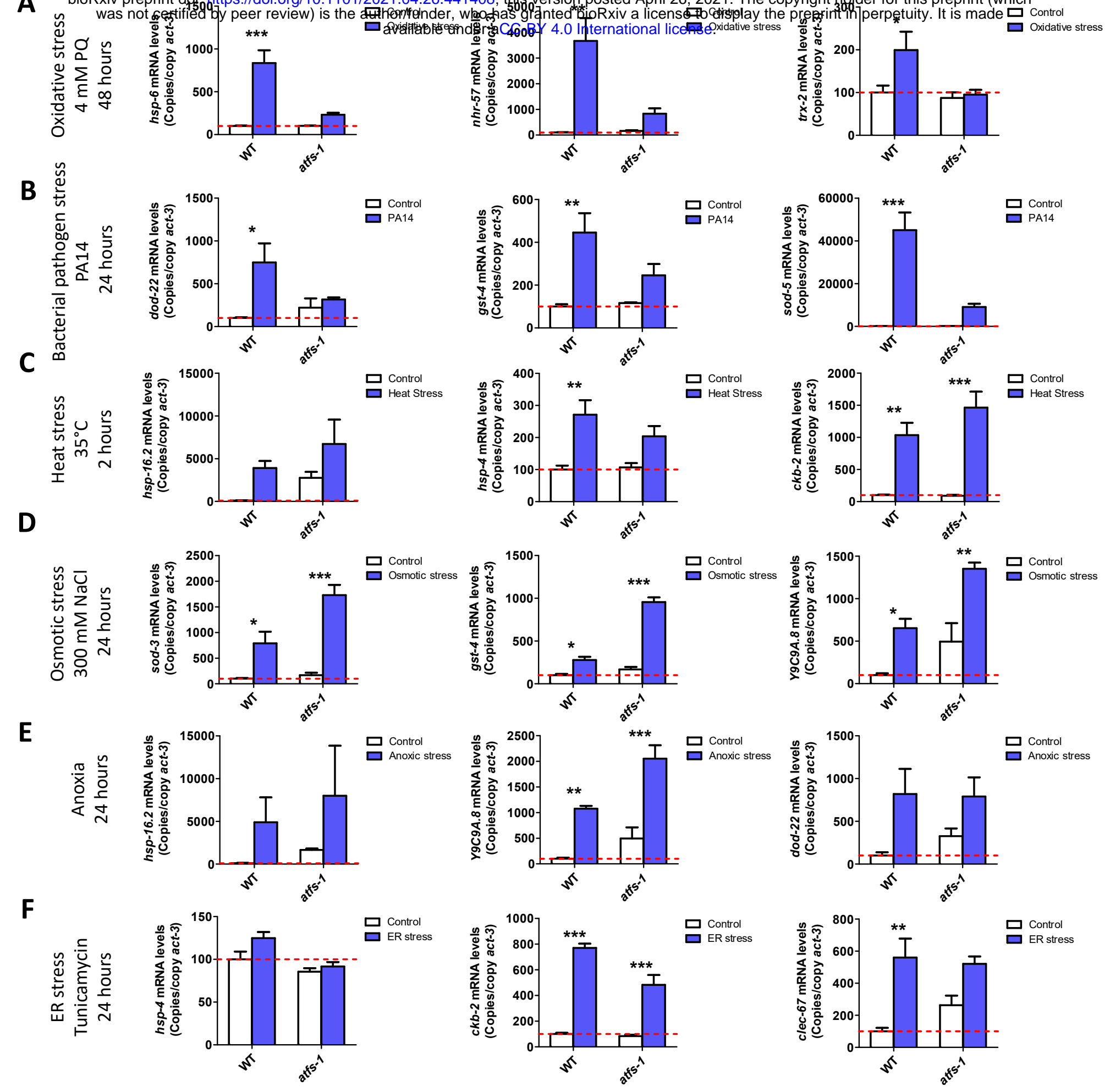

$\square$ Oxidative stress

Figure 3. ATFS-1 is required for upregulation of stress response genes after exposure to oxidative stress or bacterial pathogen stress. To determine the role of ATFS-1 in responding to different types of stress, we compared the upregulation of stress response genes in wild-type and atfs-1(gk3094) loss-of-function deletion mutants after exposure to different stressors. A. Exposure to oxidative stress ( $4 \mathrm{mM}$ paraquat, 48 hours) caused a significant upregulation of $h s p-6, n h r-57$ and $t r x-2$ in wild-type worms that was prevented by the disruption of atfs-1. B. Exposure to bacterial pathogen stress (PA14, 24 hours) resulted in an upregulation of dod-22, gst-4 and sod-5 in wild-type worms that was prevented by the atfs-1 deletion. C. Exposure to heat stress $\left(35^{\circ} \mathrm{C}, 2\right.$ hours) increased the expression of $h s p-16.2, h s p-4$ and $c k b-2$ in both wildtype and atfs-1 worms. D. Exposure to osmotic stress (300 mM, 24 hours) caused an upregulation of sod-3, gst-4 and Y9C9A.8 in wild-type worms and to a greater magnitude in atfs-1 mutants. E. Anoxia (24 hours) resulted in the upregulation of hsp-16.2, Y9C9A.8 and dod-22 in both wild-type and atsf-1 worms. F. Exposing worms to endoplasmic reticulum stress (tunicamycin, 24 hours) increased the expression of $c k b-2$ and clec-67 in both wild-type and atfs-1 worms. Error bars indicate SEM. ${ }^{*} p<0.05,{ }^{*} p<0.01, * * * p<0.001$. 

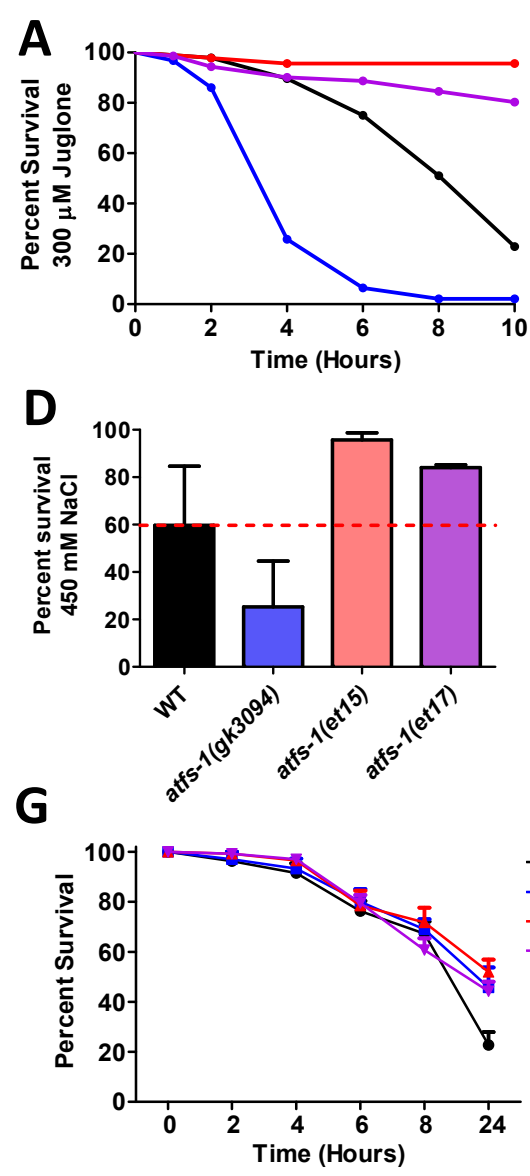

B
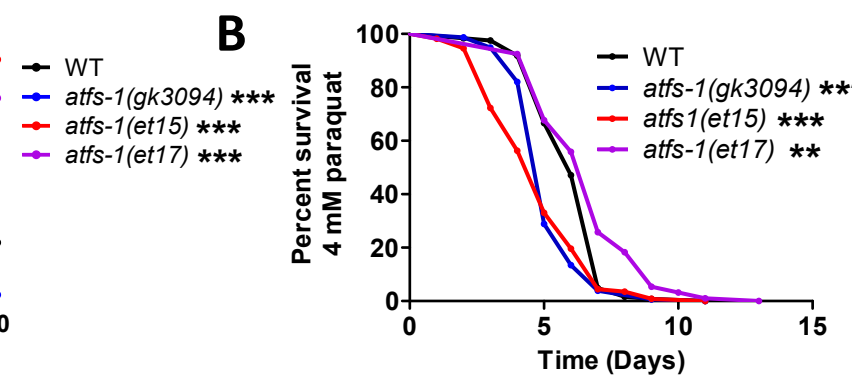

E

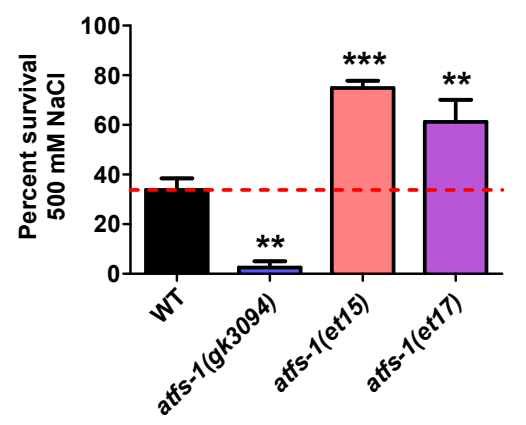

H

- WT

$\because$ atfs-1 $(g k 3094) * * *$

- atfs-1(et-15) ***

$\rightarrow$ atfs-1(et-17) **
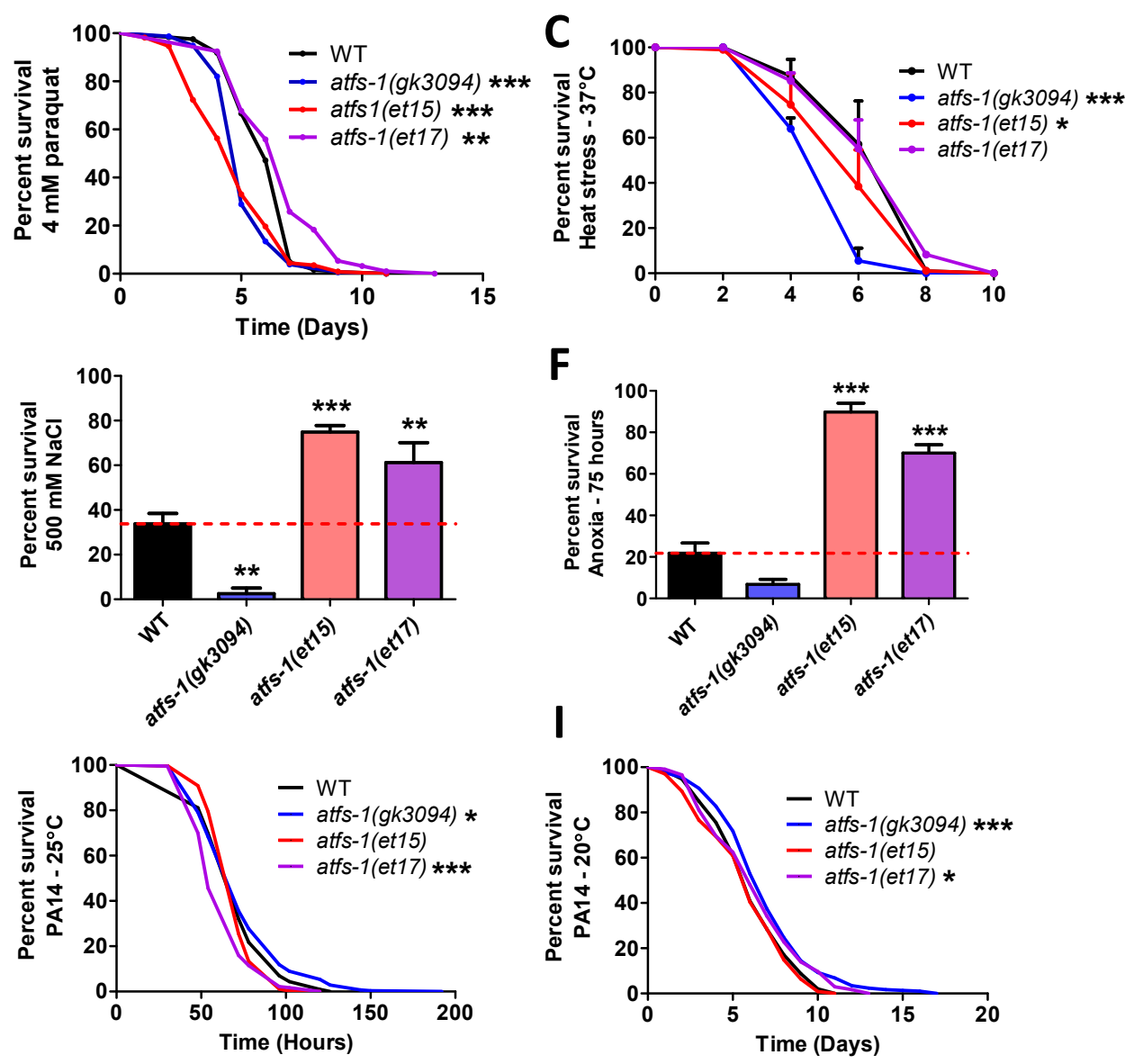

Figure 4. Constitutive activation of ATFS-1 increases resistance to multiple external stressors. To determine the role of ATFS-1 in resistance to stress, the stress resistance of an atfs-1 loss-of-function mutants (atfs-1(gk3094)) and two constitutively active atfs-1 gain-of-function mutants (atfs-1(et15), atfs-1(et17)) was compared to wild-type worms. A. Activation of ATFS-1 enhanced resistance to acute oxidative stress ( $300 \mu \mathrm{M}$ juglone), while deletion of atfs-1 markedly decreased resistance to acute oxidative stress. B. Disruption of atfs-1 decreased resistance to chronic oxidative stress (4 mM paraquat). atfs-1(et17) mutants showed increased resistance to chronic oxidative stress, while atfs1(et15) mutants had decreased resistance. C. Resistance to heat stress $\left(37^{\circ} \mathrm{C}\right)$ was not enhanced by activation of ATFS-1, while deletion of atfs-1 decreased heat stress resistance. D,E. Activation of ATFS-1 increased resistance to osmotic stress ( $450 \mathrm{mM}, 500 \mathrm{mM} \mathrm{NaCl})$, while disruption of atfs-1 decreased osmotic stress resistance. F. Constitutively active atfs-1 mutants show increased resistance to anoxia (75 hours), while atfs-1 deletion mutants exhibit a trend towards decreased anoxia resistance. G. Activation of ATFS-1 increased resistance to $P$. aeruginosa toxin in a fast kill assay. A slow kill assay in which worms die from internal accumulation of $P$. aeruginosa was performed according to two established protocols. H. At $25^{\circ} \mathrm{C}$, atfs-1(et17) mutants showed a small decrease in resistance to bacterial pathogens (PA14), while atfs-1(gk3094) mutants showed a small increase in resistance. I. At $20^{\circ} \mathrm{C}$, both atfs-1(et17) and atfs-1(gk3094) mutants exhibited a small increase in resistance to bacterial pathogens. Error bars indicate SEM. ${ }^{*} p<0.05, * * p<0.01$, $* * * p<0.001$. 


\section{clk-1}

265101737

6.7 fold enrichment $28 \%$ overlap; $p=1.7 \times 10^{-55}$

nuo-6

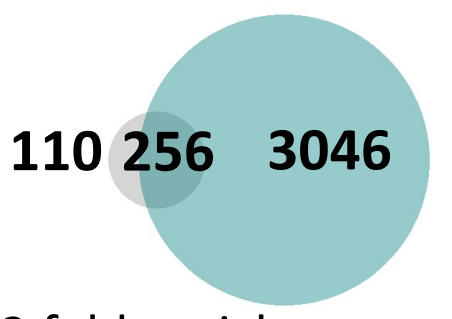

4.3 fold enrichment $70 \%$ overlap; $p=2.8 \times 10^{-119}$ isp-1

2011651344

6.1 fold enrichment

$45 \%$ overlap; $p=2.6 \times 10^{-89}$

daf-2

2381282456

2.8 fold enrichment

$35 \%$ overlap; $p=7.5 \times 10^{-29}$

sod-2

32937331

5.6 fold enrichment $10 \%$ overlap; $p=1.7 \times 10^{-17}$

\section{glp-1}

2341323289

2.2 fold enrichment

$36 \%$ overlap; $p=1.6 \times 10^{-19}$

eat-2

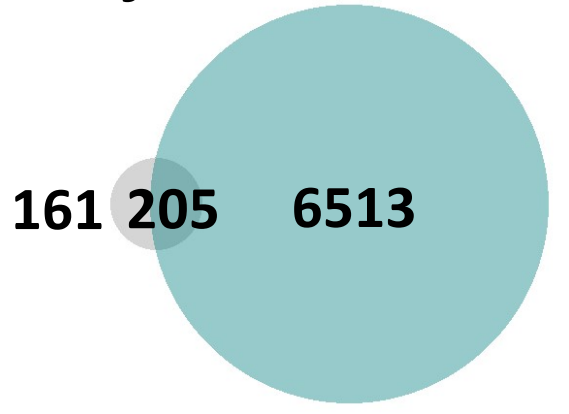

1.7 fold enrichment $56 \%$ overlap; $p=2.7 \times 10^{-20}$ osm-5

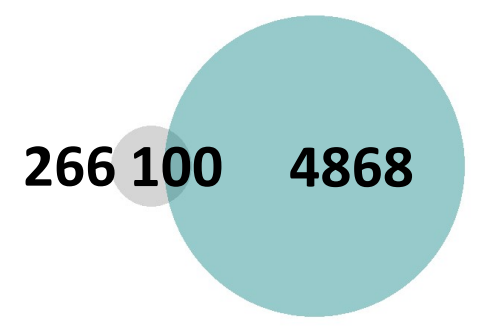

No enrichment

$27 \%$ overlap; $p=N S$

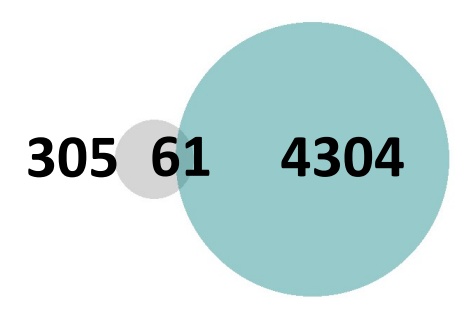

No enrichment

$17 \%$ overlap; $p=0.015$

\section{Figure 5. Multiple long-lived mutants from different pathways of lifespan extension show} upregulation of ATFS-1-dependent genes. To determine the extent to which long-lived genetic mutants from different pathways of lifespan extension show differential expression of ATFS- 1 target genes, we compared genes that are upregulated in nine different long-lived mutants to a published list of $s p g-7$ RNAi-upregulated, ATFS-1-dependent target genes (Nargund et al., 2012). We found that $c l k-1$, isp-1, nuo-6, sod-2, daf-2, glp-1 and ife-2 worms all show a highly significant degree of overlap with genes upregulated by spg-7 RNAi in an ATFS-1-dependent manner. The grey circles represent the 366 genes that are upregulated by spg-7 RNAi in an ATFS-1 dependent manner. Turquoise circles are genes that are significantly upregulated in the long-lived mutant indicated as determined from our RNA sequencing data. The number of unique and overlapping genes are indicated. Overlap is calculated as the number of genes in common between the two gene sets divided by the total number of genes that are upregulated by spg-7 RNAi in an ATFS-1 dependent manner. Enrichment is calculated as the number of overlapping genes observed divided by the number of overlapping genes predicted if genes were chosen randomly. 


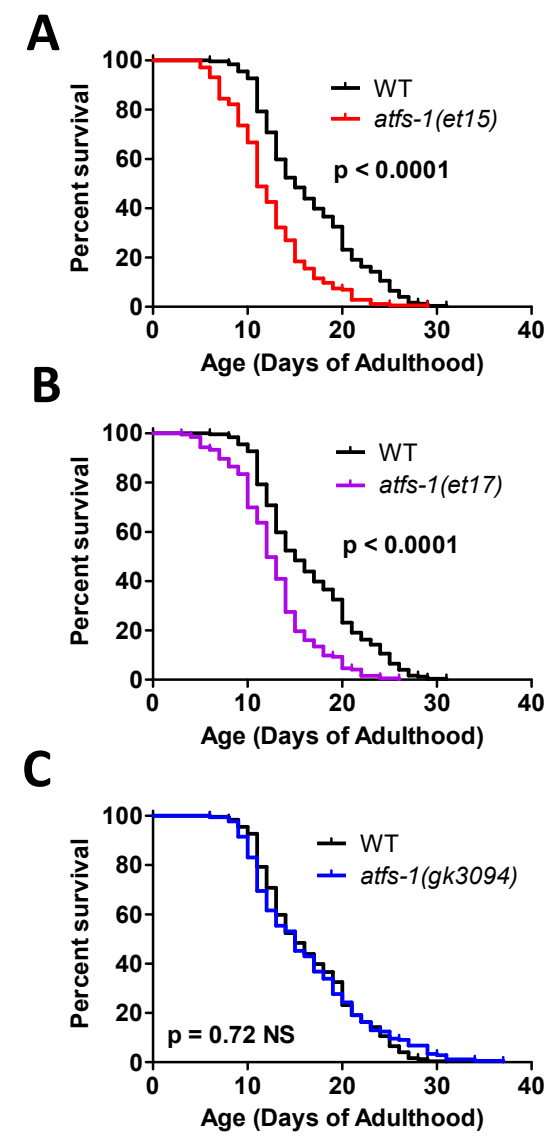

Figure 6. Activation of ATFS-1 does not increase lifespan. To determine the effect of ATFS-1 on aging, we quantified the lifespan of an atfs-1 deletion mutant and two constitutively active atfs-1 mutants. A,B. Both constitutively active atfs-1 mutants, et 15 and et17, have a significantly decreased lifespan compared to wild-type worms. C. Deletion of atfs-1 does not affect lifespan compared to wild-type worms. atfs-1(gk3094) is a loss of function mutant resulting from a deletion. atfs-1(et15) and atfs-1(et17) are constitutively active gain-of-function mutants. 
Supplementary Figures for:

\section{Mitochondrial unfolded protein response transcription factor ATFS-1 increases resistance to exogenous stressors through upregulation of multiple stress response pathways}

Sonja K. Soo ${ }^{1,2}$, Annika Traa ${ }^{1,2}$, Meeta Mistry³, Jeremy M. Van Raamsdonk ${ }^{1,2,4,5}$

${ }^{1}$ Department of Neurology and Neurosurgery, McGill University, Montreal, Quebec, Canada

${ }^{2}$ Metabolic Disorders and Complications Program, and Brain Repair and Integrative Neuroscience Program, Research Institute of the McGill University Health Centre, Montreal, Quebec, Canada ${ }^{3}$ Bioinformatics Core, Harvard School of Public Health, Harvard Medical School, Boston. MA, USA ${ }^{4}$ Division of Experimental Medicine, Department of Medicine, McGill University, Montreal, Quebec, Canada

${ }^{5}$ Department of Genetics, Harvard Medical School, Boston, MA, USA 
2151754755

3.5 fold enrichment $50 \%$ overlap; $p=8.9 \times 10^{-267}$ nuo-6

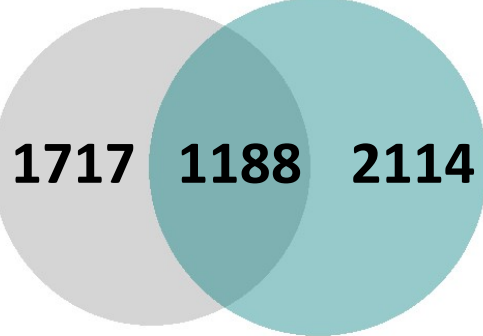

2.5 fold enrichment $41 \%$ overlap; $p=1.6 \times 10^{-272}$
$2511394 \quad 444$

3.3 fold enrichment $47 \%$ overlap; $p=2.5 \times 10^{-121}$

daf-2

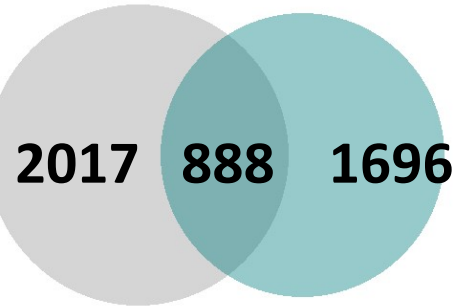

2.4 fold enrichment $34 \%$ overlap; $p=6.3 \times 10^{-175}$

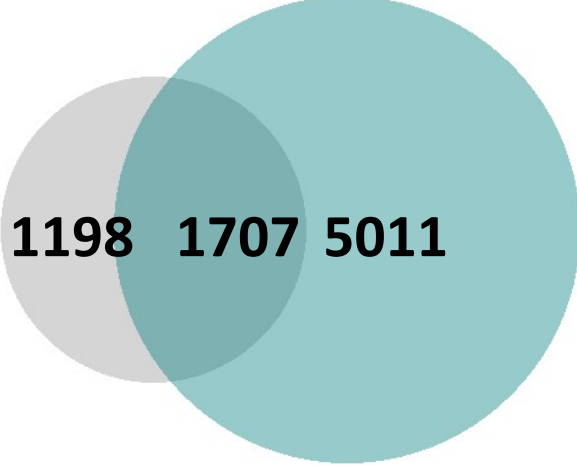

1.8 fold enrichment $59 \%$ overlap; $p=4.9 \times 10^{-213}$ eat-2

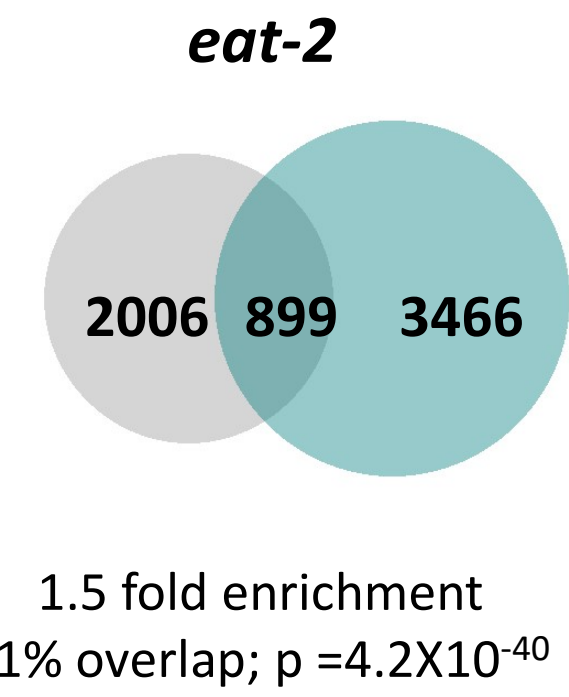

2729176192 $48 \%$ overlap; $p=7.6 \times 10^{-55}$

glp-1

$\begin{array}{lll}2021 & 884 & 2537\end{array}$

Figure S1. Multiple long-lived mutants from different pathways of lifespan extension show upregulation of ATFS-1-dependent genes. To determine the extent to which long-lived genetic mutants from different pathways of lifespan extension show differential expression of ATFS-1 target genes, we compared genes that are upregulated in nine different long-lived mutants to genes upregulated in a constitutively active atfs-1 mutant (et15). All of the long-lived mutant worms, except for osm-5, show a highly significant degree of overlap with the constitutively active atfs-1 mutant. The grey circles represent genes that are significantly upregulated in the constitutively active atfs-1(et15) mutant. Turquoise circles are genes that are significantly upregulated in the long-lived mutant indicated. The number of unique and overlapping genes are indicated. Overlap is calculated as the number of genes in common between the two gene sets divided by the smaller gene set. Enrichment is calculated as the number of overlapping genes observed divided by the number of overlapping genes predicted if genes were chosen randomly. 


\section{Table S1. Target genes examined for each stress response pathway.}

\section{Target Gene}

hsp-6

hsp-4

hsp-16.2

sod-3

gst-4

$n h r-57$

Y9C9A.8

$\operatorname{tr} x-2$

\section{Stress response pathway}

Mitochondrial unfolded protein response

ER unfolded protein response

Cytoplasmic unfolded protein response

DAF-16-mediated stress response pathway

SKN-1-mediated stress response pathway

HIF-1-mediated hypoxia pathway

p38-mediated innate immune pathway

Antioxidant genes 


\section{Table S4. Effect of modulating ATFS-1 levels and activation on stress resistance, lifespan and expression of stress response genes.}

\begin{tabular}{l|c|c|c} 
& $\begin{array}{c}\text { atfs-1(et15) } \\
\text { Gain-of-function }\end{array}$ & $\begin{array}{c}\text { atfs-1(et17) } \\
\text { Gain-of-function }\end{array}$ & $\begin{array}{c}\text { atfs-1(gk3094) } \\
\text { Loss-of-function }\end{array}$ \\
\hline Acute oxidative stress resistance & $\uparrow$ & $\uparrow$ & $\downarrow$ \\
\hline Chronic oxidative stress resistance & $\downarrow$ & $\uparrow$ & $\downarrow$ \\
\hline Heat stress resistance & $\downarrow$ & $=$ & $\downarrow$ \\
\hline Osmotic stress resistance & $\uparrow$ & $\uparrow$ & $\downarrow$ \\
\hline Anoxia resistance & $\uparrow$ & $\uparrow$ & $\downarrow$ \\
\hline Bacterial pathogens resistance & $==$ & $\downarrow \uparrow$ & $=$ \\
\hline Lifespan & $\downarrow$ & $\downarrow$ & $=$ \\
\hline hsp-6 expression (mitoUPR) & $\uparrow$ & $\uparrow$ & $=$ \\
hsp-4 expression (ER-UPR) & $=$ & $=$ & $\uparrow$ (NS) \\
hsp-16.2 expression (Cyto-UPR) & $\uparrow(\mathrm{NS})$ & $\uparrow(\mathrm{NS})$ & $=$ \\
sod-3 expression (DAF-16) & $\uparrow$ & $\uparrow$ & $=$ \\
gst-4 expression (SKN-1) & $=$ & $=$ & $=$ \\
nhr-57 expression (HIF-1) & $\uparrow$ & $=$ & $=$ \\
Y9C9A.8 expression (Innate immunity) & $\uparrow$ & $\uparrow$ & $=$ \\
trx-2 (antioxidant) & $\uparrow$ & $\uparrow$ &
\end{tabular}

